corresponding sequence from rapl GAP, which appears superfically to be the most similar in this region to $p n$ according to Fig. $3 d$ of ref. 1, no significant alignment was produced; in fact neither did the rap1 GAP subsequence when compared to the Ras GAP subsequences (contrary to the implications of Fig. $3 d$ ). Indeed, it has also been the impression of others that rap1 GAP is not significantly similar to any other protein, either compared globally, or using this subsequence ${ }^{8}$.

Consequently, we find that there is no similarity between $p n$ and GAPS - pn is as likely to be a GAP as is any randomly chosen protein. In the absence of confirmatory biochemical or genetic data concerning the model proposed by Teng et al. ${ }^{1}$ must therefore be regarded currently as untenable. Because their model fails to explain the known biochemical defect in eye pigment production in $p n$ flies ${ }^{9.10}$, the simplest and not very unusual explanation would be that $p n$ encodes a novel protein of unknown function.

THOMAS M. BARNES

THOMAS R. BÜRGLIN

Department of Molecular Biology,

Massachusetts General Hospital,

and Department of Genetics,

Harvard Medical School, Boston,

Massachusetts 02114, USA

VENKatesh AND TENG REPLy - It is evident using computer comparison programs that the deduced TcD37 protein of Drosophila does not exhibit a high degree of homology with GAP or GAP-like proteins and might therefore be a novel protein with an unknown function. However, by these very same criteria (as noted by Barnes and Bürglin and others ${ }^{8}$ ), computer analysis would

1. Teng, D. H. F. et al. Nature 353, 437-440 (1991).

2. Ruggieri, R. \& McCormick. F. Nature 353, 390 (1991).

3. Biggs, J. et al. Cell $63.933-940$ (1990).

3. Biggs, J. et al. Cell 63. $933-940$ (1990).
4. Devereux, J. et al. Nucleic Acids Res. 12, 387-395 Devereux,
(1985).

5. Pearson, W. R. \& Lipman. D. J. Proc, natn. Acad. Sci. U.S.A. 85, 2444-2448 (1988)

6. Altschul, S. F. et al. J. molec. Biol. 215, 403 (1990)

7. Protein Identification Resource (National Biomedical Research Foundation, Georgetown University Medical Center, Washington, DC 20007)

8. Rubinfeld, B. et al. Cell 65, 1033-1042 (1991)

9. Evans, B. A. \& Howelis. A. J. Biochem. Genet. 16, 13-26 (1978).

10. Mackay. W. J. \& O'Donnell, J. Genetics 105, 35-53 (1983)

11. Tanaka, K., Lin. B. K., Wood, D. R. \& Tamanoi, F. Proc natn. Acad. Sci. U.S.A. 88, 468-472 (1991)

2. Vogel, U. S et al. Nature 335, 90-93 (1988)

12. Vogel, U. S. et al. Nature 335, $90-93$ (198.

14. Colicelli, J. et al. Proc. natn. Acad. Sci. U.S.A. 88, 2913-2917 (1991).

15. Teng. D. H. F. et al. Genetics 128, 373-380 (1991)

16. Liotta. L. A. \& Steeg, P. S. J. natn. Cancer Inst. 82, 1170-1172 (1990)

17. Liotta, L. A. Steeg, P. S. \& Stetler-Stevenson, W. G. Cell 64, 327-336 (1991)

18. Kimura, N. \& Johnson, G. S. J. biol. Chem. 258 $12609-12617$ (1983)

19. Uesaka, H., Yokoyama. M. \& Othsuki, K. Biochem. Biophys. Res. Commun. 143, 552-559 (1987),

20. Othsuki, K. \& Yokoyama. M. Biochem. Biophys. Res. Commun. 148, 300-307 (1987).

21. Randazzo, P. A., Northup, J. K. \& Kahn, R. A. Science 254, $850-853$ (1991) not predict that mammalian rap1 GAPs are related to Ras GAPs. Yet both of these subclasses of proteins have been shown to function as GTPase activating proteins ${ }^{8,11-13}$. In addition, other proteins, such as the products of JC99 and JC265, which have marginal similarity to GAPs also appear to participate in the biochemical pathway involving $\operatorname{Ras}^{14}$. Thus, based on the available sequences of GAP and GAP-like proteins, it seems that these proteins are quite divergent and that the assessment of their function solely on the basis of significant homology would be inadequate.

The model we have proposed to explain the lethal interaction between $p n$ and $a w d^{k-p n}$ is not only based on sequence similarity but also draws upon our current understanding of these two loci. The lethality of $p n a w d^{K-p n}$ double mutants is apparently due to the expression of $\mathrm{Awd}^{\mathrm{K}-\mathrm{pn}}$ in combination with a lack of Pn (ref. 15). The awd locus encodes an NDP kinase ${ }^{3}$ and $a w d^{K-p n}$ is a missense mutation that appears to yield a protein product with a neomorphic function (A. Shearn, personal communication). It has been suggested that NDP kinases (like Awd and Nm23) regulate some critical biological processes, such as development and tumour metastasis, via a GTP-binding protein ${ }^{16,17}$. This hypothesis is supported by studies that implicate NDP kinases in the effector activation of certain $G$ proteins ${ }^{18-20}$, and three different NDP kinases can activate the small $G$ protein, ADPribosylation factor (Arf), by the direct phosphorylation of Arf-GDP to Arf-

\section{Are Anolis lizards evolving?}

SIR - Since Hurlbert' ${ }^{\prime}$ published his than in other enclosures), or average paper on experimental design in 1984, ecologists have been particularly careful to replicate their experiments. However, experiments without adequate replication are still occasionally performed, and the recent report of selection in Anolis lizards ${ }^{2}$ is an example. This experiment involved measuring differential survival of lizards with respect to their morphology. Morphologically distinct lizards from four populations from different habitats (ecotypes) were maintained in separate enclosures in one habitat. There was significant differential survival of both males and females in just one of the enclosures. Because each ecotype was maintained in only one enclosure there was no replication within ecotypes. The key result was that selection (differential survival) occurred in the ecotype derived from the most distinct habitat.

The significant selection in the one enclosure could be due to this ecotypic difference, as the authors argue, but it may also be due to density (much lower
GTP (ref. 21). Furthermore, contrary to Barnes and Bürglin's statement that Awd is principally microtubuleassociated, immunological and biochemical studies indicate that only a small proportion of the enzyme appears to be associated with cytoskeleton while the most of it is found in other subcellular locations, such as the cytosol and nucleus (A. Shearn, personal communication). Because much of the nonmicrotubule-associated NDP kinase is probably also Awd protein (as homozygous awd mutants have less than $2 \%$ of the total enzyme activity of wild-type larvae ${ }^{3}$ ), this subcellular distribution suggests that Awd may provide NTPs for more than one biological process. In the light of this information, we have proposed a new model as one possible explanation of the $p n a w d^{K-p n}$ lethality. As with any model, its validity is subject to the rigours of testing by molecular, biochemical and genetic approaches.

Finally, Barnes and Bürglin state that our model fails to account for the pigment biosynthesis defect in the eyes of $p n$ flies. But, as noted by Ruggieri and McCormick $^{2}$, it is conceivable that the hypothetical Ras-like $\mathrm{G}$ protein modulated by Awd and $\mathrm{Pn}$ is also involved in regulating the biosynthesis of pteridine pigments in Drosophila.

TADMIRI R. VENKATESH*† DAVID H. F. TENG*

Institutes of Molecular Biology* and Neurosciencet,

and Department of Chemistryt. University of Oregon,

Eugene, Oregon 97403, USA body size (much higher than in other enclosures). More seriously, it could be random. This can be illustrated using a thought experiment, such as the following 'fox-test': if one rogue fox that ate lizards and led to differential survival of different morphologies is dropped into the experiment at random, one-quarter of the time it will land in the enclosure that contains the ecologically most different morphotype, and all results follow. The probability of the association between intense selection and location is thus 0.25 , which is clearly not significant. By this 1 do not mean to imply that foxes are the selective agent, but rather that something peculiar to one enclosure can cause the apparent selection. Replication of treatments over experimental units is the only way to estimate and control for such random variation. Inferential statistics such as ANOVA, which test for treatment effects, require an estimate of error within treatments. In this case, the treatment variable is ecotype, for which there is no replication (only one enclo- 
sure for each ecotype). The authors note that their study is a long-term experiment. Without alteration of the experimental design, future results may be suspect.

Department of Biology,

University of California, San Diego,

La Jolla, California 92093, USA

SIR - Malhotra and Thorpe ${ }^{2}$ provide no evidence for a rapid evolutionary response in natural lizard populations. Their experiment, which is conceptually ingenious and potentially important, was based on the translocation of four populations of Anolis oculatus from different ecosystems to four neighbouring arenas. The authors claim that there was rapid selective mortality in the translocated populations, and that this is evidence for the action of natural selection.

There are three problems with their experiment. (1) The design is what statisticians call confounded and ecologists pseudoreplicated. Because each population is in only one arena it is impossible to tell whether the claimed effect is due to differences between arenas or between populations.

(2) The correlation coefficient quoted in Fig. 2 is not statistically significant. There are not six degrees of freedom (as the authors appear to assume) but only two (there being four arenas). However, the figure does show a large response in one deviant population. As this montane population was from the ecologically most distinct site it could still support the authors' conclusion that the differential mortality is evidence of rapid evolutionary change due to natural selection.

(3) Unfortunately, even if we accept that the effect is due to the montane population responding differently to translocation, this does not necessarily imply natural selection. Using the values in Table 1 of ref. 2 we can calculate the average weight of the lizards placed in the different arenas: control $3.76 \mathrm{~g}, \mathrm{~S}$. Caribbean $4.6 \mathrm{~g}$, Atlantic $5.26 \mathrm{~g}$, Montane $8.46 \mathrm{~g}$. Lizards from the montane population were, on average, more than twice the weight of the controls when they were translocated. Because the morphological measurements taken have

1. Hurlbert, S. H. Ecol. Monogr. 54, 187-211 (1984).

2. Malhotra, A. \& Thorpe, R S Nature $353,347-348$ (1991).

3. Atchley, W. R., Rutledge, J. J. \& Cowley, D. E. Evolution 35, 1037-1055 (1981)

4. Atchley, W. R. in Numerical Taxonomy (ed. Felsenstein, J.) 346-363 (NATO Advanced Study Institute Series G, Springer, Berlin, 1983)

5. Falconer, D. S. Introduction to Quantitative Genetics (Longman, London, 1981)

6. Losos, J. Evolution 44, 55-569 (1990).

7. Thorpe, R. S. J. Zool. Lond. 216, 37-40 (1988).

8. Thorpe, R. S. Syst. zool. 40, 172-187 (1991).

9. Brown, R. P., Thorpe, R. S. \& Baez, M. Nature 352 , 60-62 (1991)

10. Manly, B. J. F. Randomization and Monte Carlo Methods in Biology (Chapman and Hall, London, Methods
1991). not been corrected for body size or age, there is the strong possibility that it was the larger or older animals that died. No evidence is presented that the difference in body size between the populations is genetically based. We therefore reluctantly conclude that no reliable evidence of a rapid evolutionary respone has been presented.

J. H. LAWTON B. H. MCARDLE NERC Centre for Population Biology, Imperial College, Silwood Park, Ascot, Berkshire SL5 7PY, UK

ThORPE AND MALHOTRA REPLy - Our study $^{2}$ shows clear evidence of rapid evolutionary response in lizards and Katti's and Lawton and McArdle's claims to the contrary are based on flawed logic and misunderstanding of the statistics used.

In particular, they reach their erroneous conclusions because they fail adequately to differentiate between the within-enclosure study of morphology, which supplied the critical evidence, and the between-enclosure study of 'fitness', which supplied only supplementary information. Their point that the difference between ecotypes could be due to chance differences between enclosures cannot pertain to the difference in the morphology of survivors and nonsurvivors within an enclosure, but only to the between enclosure analysis of 'fitness'.

Katti's claim that the "differential survival" of ecotypes could be due to density, average body size, or chance might have been true if we had measured differential survival as the relative number of survivors in each ecotype, but we did not do this. We measured the difference in morphology between survivors and non-survivors, and the fact that the most foreign ecotype exhibited a significant difference within an enclosure has to be due to differential selection, irrespective of any differences between enclosures, be they chance, average body size or density. Even so, biomass was constant between enclosures and density was lowest in the enclosure with greatest selection effects. Lawton and

\section{Scientific Correspondence}

SCIENTIFIC Correspondence is a relatively informal section of Nature in which matters of general scientific interest, not necessarily those arising from papers appearing in Nature, are published. Due to space limitations priority is usually given according to general interest and topicality, to contributions of fewer than 500 words, and to contributions using simple language. Contributions may be sent to referees and, in the case of matters arising from material published in Nature, are often sent to the author of that article for comment.
McArdle's first point is rejected for the morphology on the same basis. The point regarding replicaton is valid for the fitness variables, but we are dealing here not with a small plot of ground in a suburban research station, but with about $100,000 \mathrm{~m}^{3}$ of remote natural forest per set of enclosures, requiring constant management. For several replications, the time required is prohibitive and the environmental perturbation caused is unacceptable, given the entirely supplementary and non-critical role of these fitness data. Moreover, a replicate in the Atlantic habitat supports our earlier results in showing a significant difference in morphology between survivors and non-survivors of foreign ecotypes and their lower fitness (growth rate, condition and weight change).

The average specimen size differs among ecotypes because size varies racially and not because of sample bias. There is no trend for preferential survival of a given size within an enclosure. There is no basis for excluding a single measure of size as it tends to be as highly heritable as other characteristics ${ }^{3-6}$. 'Size' has to be taken into account because of its intercorrelating effect, and this we do properly by using Mahalonabis $D^{2}$ (refs 7,8) as Lawton and McArdle should have realized. Consequently, the significant difference between montanetype survivors/non-survivors is unaltered by the exclusion of snout-vent length and/or size-adjusting the few linear measurements. This is the most important point because, as Lawton and McArdle admit, this significant difference is sufficient to prove our case.

With regard to the correlation between the dissimilarity in ecology and morphological dissimilarity between survivors/non-survivors, Lawton and McArdle do not appear to regard the $D^{2}$ for sexes as varying independently, even though it clearly does so. Random permutation techniques ${ }^{8-10}$ which give the probability of association when the data points are not independent, indicate a significant association $(P<0.01,10,000$ permutations). Moreover, if one accepts Lawton and McArdle's point, the morphological dissimilarity can be averaged across sex (giving four points) and the relationship linearized. This gives a correlation of $r=1.0000$ which is significant whatever degrees of freedom are used.

Our morphological analysis provides sound evidence of rapid evolution in Anolis oculatus and our analysis of fitness, although non-critical, provides interesting information on the interface between evolution and ecology.

\section{R. S. THORPE}

A. Malhotra

Department of Zoology,

University of Aberdeen,

Aberdeen AB9 2TN, UK

NATURE · VOL $355 \cdot 6$ FEBRUARY 1992 